\title{
Lesión quística testicular en un lactante: consideraciones diagnósticas y terapéuticas
} Considerations

Recepción: 11/02/2019 | Aceptación: 07 Junio 2019

\author{
Laura González Villarreal \\ Médica cirujana, Pontificia Universidad Javeriana, \\ Bogotá, Colombia \\ Fernando Augusto Escobar Rivera a \\ Médico especialista en cirugía pediátrica. Coordinador \\ del Servicio de Cirugía Pediátrica, Hospital San Rafael \\ de Tunja, Colombia \\ Vicente de Jesús Aljure Reales \\ Médico especialista en radiología. Coordinador \\ del Departamento de Radiología e Imágenes \\ Diagnósticas, Hospital San Rafael de Tunja. \\ Profesor asociado de la Universidad Pedagógica y \\ Tecnológica de Colombia y Universidad de \\ Boyacá Tunja, Colombia
}

a Correspondencia: lgonzalez.v@javeriana.edu.co

\footnotetext{
Cómo citar: González Villarreal L, Escobar Rivera FA, Aljure Reales VJ. Lesión quística testicular en un lactante: consideraciones diagnósticas y terapéuticas. Univ. Med. 2019;60(4). https:// doi.org/10.11144/Jave riana.umed60-4.lqtl
}

\section{RESUMEN}

Introducción: Los tumores testiculares prepuberales suelen ser benignos. Las lesiones quísticas constituyen un amplio diagnóstico diferencial de las masas benignas escrotales en niños. Una adecuada evaluación incluye el examen clínico, la ecografía Doppler y la medición de marcadores tumorales. Ante una fuerte sospecha clínica y radiológica de benignidad, el tratamiento de elección es la enucleación con preservación del parénquima. Objetivo: Describir el caso de un lactante con una masa quística de testículo compatible con quiste epidermoide. Discutir los aspectos clínicos, imagenológicos y terapéuticos más relevantes de las lesiones testiculares prepuberales. Presentación del caso: Un lactante ingresa por identificación de una masa testicular circunscrita no dolorosa de $1 \mathrm{~cm}$ de diámetro. La ecografía demuestra un quiste simple intraparenquimatoso y los marcadores tumorales son negativos. Se realiza enucleación por vía escrotal, sin complicaciones. El estudio histopatológico es compatible con un quiste epidermoide. Hubo seguimiento a 3 años sin recidiva ni atrofia testicular. Discusión y conclusión: Teniendo en cuenta que la mayoría de los tumores prepuberales de testículo son benignos, la cirugía conservadora puede considerarse una alternativa terapéutica ante una lesión circunscrita, pequeña, avascular, con marcadores tumorales negativos.

Palabras clave

quiste epidermoide; neoplasias testiculares; niños; ultrasonografía Doppler.

\begin{abstract}
Introduction: Prepuberal testicular tumors are usually benign. Cystic lesions constitute a wide group of scrotal benign masses differentials in children. An appropriate evaluation comprises a complete clinical exam, Doppler ultrasonography and tumor markers. If a benign lesion is likely, testis-sparing surgery is the treatment of choice. Objective: To describe the case of an infant with a cystic testicular mass compatible with epidermoid cyst. Furthermore, to discuss the most relevant clinical,
\end{abstract}


diagnostic and therapeutic aspects of prepubertal testicular lesions. Case report: An infant was admitted due to identification of a painless well-defined testicular mass of approximately $1 \mathrm{~cm}$ of diameter. Ultrasonography showed an intraparenchymal simple cyst and tumor markers were negative. Complete enucleation was performed via scrotal approach without postoperative complications. Histopathological study was compatible with a testicular epidermoid cyst. There was no evidence of recurrence or atrophy at 3-year follow-up. Discussion and conclusion: Since most prepuberal testicular tumors are benign, testissparing surgery constitutes a therapeutic alternative in small, circumscribed, avascular lesions with negative tumor markers.

Keywords

epidermoid cyst; testicular neoplasms; children; Doppler ultrasonography.

\section{Introducción}

Las neoplasias testiculares constituyen del $1 \%$ al $2 \%$ de los tumores en niños y su incidencia se encuentra alrededor de 0,05 a 2 por cada 100.000 casos $(1,2,3,4)$. Suelen tener una presentación bimodal, con un primer pico de incidencia en menores de 3 años y un segundo pico en adolescentes (2). Con mayor frecuencia, los tumores prepuberales son de carácter benigno en comparación con los pospuberales $(1,5)$. Dentro de la categoría de los tumores benignos prepuberales se incluyen las lesiones quísticas, que son muy poco frecuentes en la infancia y suelen constituir un hallazgo incidental en ecografía (6).

El principal diagnóstico diferencial de una lesión quística testicular prepuberal es el teratoma, el cual suele ser de carácter benigno en este grupo de edad (7). Le sigue en frecuencia el quiste epidermoide testicular, un tumor de origen ectodérmico, revestido por epitelio escamoso estratificado, que contiene material queratinizado $(3,8,9,10)$. Es inusual en niños y representa del $1 \%$ al $2 \%$ de las masas benignas escrotales $(8,11)$. Las características ecográficas del quiste epidermoide testicular son variables según la cantidad de material queratinizado acumulado en su interior y guardan semejanza con otras lesiones quísticas simples o complejas $(8,12,13)$. Existen hallazgos ultrasonográficos más frecuentes en tumores benignos que pueden orientar el diagnóstico (14).

La evaluación preoperatoria incluye el examen clínico, la ecografía Doppler y la medición de marcadores tumorales (15). Una fuerte sospecha de lesión benigna está dada por el hallazgo de una masa pequeña menor de $2 \mathrm{~cm}$, firme, circunscrita, sin evidencia de flujo con exploración Doppler y marcadores tumorales dentro de los límites normales para la edad $(6,15)$. Históricamente, el abordaje terapéutico de las masas testiculares en niños se ha basado en el algoritmo de manejo propuesto para los adultos, dado que constituyen una enfermedad de muy baja prevalencia en la infancia (16). No obstante, en las últimas décadas la cirugía conservadora, definida como enucleación o tumorectomía con preservación del parénquima sano, se ha posicionado como el estándar de tratamiento de tumores testiculares en menores de 12 años, puesto que se ha descrito una tasa de benignidad cercana al 80\% $(16,17)$. En casos de lesiones quísticas que se presumen benignas, el abordaje quirúrgico de elección es la enucleación $(2,18)$, dado el mínimo riesgo de recidiva, metástasis o atrofia residual $(8,11,19,20,21,22)$.

El objetivo del presente trabajo es describir el caso de un lactante con una lesión quística testicular sometido a enucleación con preservación del parénquima, con diagnóstico histopatológico de quiste epidermoide. Este caso contribuye a entender el abordaje diagnóstico y terapéutico de las masas escrotales prepuberales, resaltando los aspectos más relevantes del manejo conservador.

\section{Presentación del caso}

El caso corresponde a un lactante de 9 meses sin antecedentes clínicos, llevado a consulta por primera vez por presentar una masa testicular de 7 meses de evolución, debido al aumento progresivo del tamaño. En el examen físico se encontró una lesión circunscrita en el testículo derecho, de bordes bien definidos, de aproximadamente $1 \mathrm{~cm}$ de diámetro, no dolorosa, sin cambios inflamatorios locales. La ecografía 
evidenció un quiste simple intraparenquimatoso (figura 1a). Los marcadores tumorales fueron negativos.

\section{Figura 1}

a) Ecografía preoperatoria: imagen anecoica, unilocular, circunscrita, de 19,2 × 11,5 × 17,1 $\mathrm{mm}$ (volumen calculado 2,0 ml). b) Ecografía postoperatoria: testículo derecho de aspecto homogéneo de 13,3 × 6,3 × 10,6 mm (volumen calculado 0,5 ml)
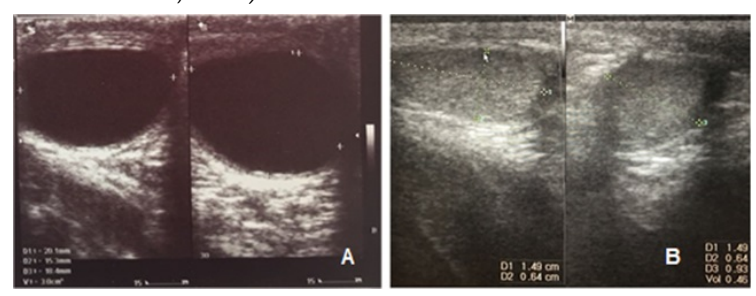

Ante los hallazgos clínicos, paraclínicos y ecográficos altamente sugestivos de quiste simple sintomático, se decidió llevar a exploración testicular por vía escrotal. Se identificó un gran quiste epidérmico que ocupaba el 70\% del volumen testicular, el cual se enucleó en su totalidad respetando el parénquima residual (figura 2). No se documentaron complicaciones perioperatorias. El estudio microscópico de la pieza evidenció un quiste con pared conformada por tejido fibroconjuntivo denso y tapizado por epitelio escamoso estratificado delgado, con queratinización superficial, sin evidencia de otros elementos teratogénicos o neoplásicos, compatible con un quiste epidermoide testicular.

\section{Figura 2}

Masa quística de $2 \times 1,5 \times 1,5 \mathrm{~cm}$, blanquecina, de superficie lisa. Se realiza enucleación completa por vía escrotal respetando el parénquima residual
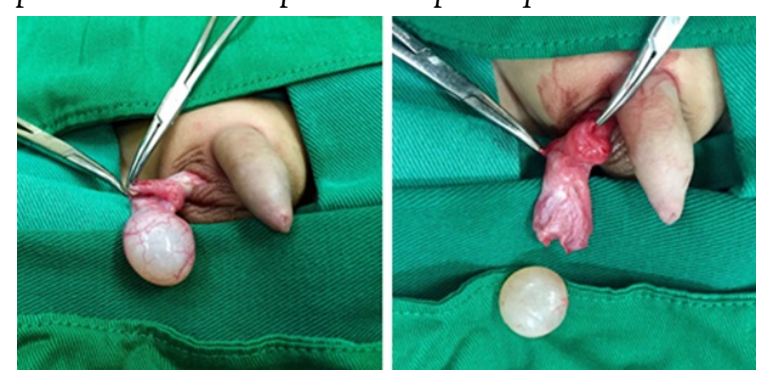

Presentó adecuada evolución postoperatoria, sin dolor ni cambios tróficos testiculares, con adecuada cicatrización de la herida. Durante el seguimiento a tres años, no se documentó recidiva. La ecografía de control mostró un adecuado volumen testicular (volumen residual del testículo derecho de 0,64 ml), sin evidencia de lesiones intraparenquimatosas (figura 1b).

\section{Discusión}

Se ha descrito que del 31\% al 74\% de las lesiones intratesticulares en niños menores de 12 años son de carácter benigno $(6,15,16,17)$. Clínicamente, son indistinguibles de una neoplasia maligna (11), dado que más del $90 \%$ de los tumores se presentan como una masa escrotal indolora (17), sólida a la palpación, que puede asociarse a hidrocele en el 15\% al 50\% de los casos $(3,7,23)$.

Existen diversos diagnósticos diferenciales de una masa testicular prepuberal. El más frecuente es el teratoma, seguido por el quiste epidermoide (7). Con menor frecuencia se observa displasia quística de la rete testis, quistes simples, quiste mesotelial vestigial, quiste dermoide, tumor de células de Sertoli, tumor de células de Leydig, fibromas, fibrolipomas y linfangiomas $(7,17)$. En recién nacidos, la lesión benigna más frecuente es el tumor juvenil de células de la granulosa $(7,24)$. Los quistes simples son infrecuentes y pueden sospecharse, como en el presente caso, ante una masa testicular en un lactante con hallazgos clínicos que orienten a etiología benigna sumado a la presencia de una lesión quística, encapsulada, sin evidencia de ecos en su interior ni flujo a la exploración Doppler (14).

De acuerdo con los criterios diagnósticos establecidos por Price (5), en 1969, desde el punto de vista histológico, el quiste epidermoide testicular se define como: 1) una lesión quística localizada en el parénquima del testículo, 2) con contenido queratinizado o material amorfo, 3) una pared compuesta por tejido fibroso con revestimiento de epitelio escamoso y 4) ausencia de componentes teratomatosos o elementos anexiales en su interior.

La apariencia ecográfica varía dependiendo del grado de madurez, tamaño y compactación de la queratina que contiene, pues se han encontrado 
lesiones quísticas hasta sólidas, lo que hace difícil su diferenciación con tumores malignos (10). De acuerdo con Taghavi y Hutson (25), los hallazgos de ultrasonido permiten clasificar el quiste epidermoide en uno de cuatro grupos: apariencia en anillo de cebolla (tipo I), masa hiperecoica con sombra acústica posterior (tipo II), masa hipoecoica con reborde bien definido (tipo III) (figura 1a) y lesión heterogénea con márgenes mal definidos y pequeños focos de calcificación (tipo IV) (26).

La presencia de una masa pequeña, firme, circunscrita, no dolorosa, con evidencia ecográfica de proceso unilocular, de contenido anecoico y bordes bien definidos, sin flujo a la exploración Doppler y marcadores tumorales negativos es muy sugestiva de lesión benigna $(7,27)$. Una fuerte sospecha diagnóstica prequirúrgica, basada en los hallazgos clínicos y radiológicos descritos, se encuentra a favor del manejo conservador $(2,9)$.

Hasta 1980, todos los tumores testiculares se presumían malignos, por lo que el manejo era, casi sin excepción, la orquiectomía radical $(7,10)$. En las últimas décadas, se ha reevaluado el abordaje terapéutico de las masas testiculares en niños, debido a la preponderancia de la enfermedad benigna en la etapa prepuberal $(3,17,28)$. Dado que la presentación clínica es muy similar para todos los casos, la evaluación diagnóstica debe incluir un examen físico completo, la ecografía Doppler testicular y la medición de marcadores tumorales (gonadotropina coriónica humana subunidad beta y alfa fetoproteína séricas) (29)

Existe controversia respecto al punto de corte de $2 \mathrm{~cm}$ para definir un tumor testicular como una lesión pequeña en pacientes pediátricos (16). En adultos se ha observado que el comportamiento de la masa se relaciona con el tamaño, por lo cual se considera aceptable el abordaje conservador en tumores menores de $2 \mathrm{~cm} \quad(16,24)$. Un estudio retrospectivo llevado a cabo por Caldwell et al. (16) evaluó 24 pacientes menores de 18 años con masas testiculares y marcadores tumorales negativos, con el fin de establecer si el punto de corte de $2 \mathrm{~cm}$ constituye un criterio adecuado para seleccionar candidatos a cirugía conservadora en la población pediátrica. Los resultados no sugieren un punto de corte significativo para establecer una patología favorable o desfavorable en niños ( $\mathrm{p}=0,312)(16)$.

En la práctica, la decisión de realizar enucleación con preservación de testículo depende en gran medida de la experiencia individual del equipo quirúrgico. Teniendo en cuenta que el porcentaje de enfermedad benigna se aproxima al $80 \%$ en menores de 12 años, la orquiectomía radical constituye un sobretratamiento en aquellos casos con una muy alta probabilidad prequirúrgica de enfermedad benigna (15).

En respeto de los principios oncológicos, se prefiere el abordaje inguinal con pinzamiento atraumático del cordón y aislamiento del campo quirúrgico, seguido de enucleación y confirmación de márgenes negativos en estudio histológico por congelación de forma intraoperatoria cuando esté disponible $(15,17,23,24,27,30)$. No obstante, similar al presente caso, existen reportes de pacientes sometidos a cirugía conservadora por vía escrotal ante el hallazgo de una lesión quística simple con diagnóstico histopatológico definitivo confirmatorio de benignidad, sin evidencia de recidiva ni metástasis $(6,31)$. A este respecto, Casaccia et al. (18) proponen que la cirugía por vía escrotal minimiza el trauma quirúrgico, lo cual sugiere que el abordaje inguinal es una maniobra innecesaria en algunos casos seleccionados.

Las ventajas del manejo conservador superan las posibles desventajas, teniendo en cuenta que la supervivencia a 5 años en tumores prepuberales se acerca al $99 \%$ (3). En una encuesta retrospectiva multicéntrica llevada a cabo en Francia, se registraron 83 lesiones benignas, con 15 quistes epidermoides de los cuales 13 fueron enucleados y 2 fueron manejados con orquiectomía. No se documentó ningún caso de recurrencia, metástasis, ni atrofia (7). Por otro lado, una cohorte retrospectiva de 67 pacientes menores de 14 años tratados por tumores testiculares entre 2005 y 2015, con 
seguimiento a 23 meses, no registró ningún caso de recurrencia ni atrofia testicular (29).

\section{Conclusión}

El presente caso ayuda a comprender el abordaje diagnóstico y terapéutico de las lesiones quísticas testiculares en niños. Teniendo en cuenta que la mayoría de los tumores prepuberales de testículo son benignos, la cirugía conservadora es una alternativa terapéutica ante una lesión circunscrita, pequeña, avascular, con marcadores tumorales negativos.

\section{Conflictos de interés}

Los autores declaran no tener ningún conflicto de interés.

\section{Referencias}

1. Friend J, Barker A, Khosa J, Samnakay N. Benign scrotal masses in children - some new lessons learned. J Pediatr Surg. 2016;51(10):1737-42. http://dx.doi.or g/10.1016/j.jpedsurg.2016.07.016

2. Ciftci AO, Bingol-Kologlu M, Senocak ME, Tanyel FC, Buyukpamukcu M, Buyukpamukcu N. Testicular tumors in children. J Pediatr Surg. 2001 Dec;36(12):1796-801.

3. Ahmed HU, Arya M, Muneer A, Mushtaq I, Sebire NJ. Testicular and paratesticular tumours in the prepubertal population. Lancet Oncol. 2010;11(5):476-83. http://dx.doi.org/1 0.1016/S1470-2045(10)70012-7

4. Docal I, Crespo C, Pardo A, Prieto A, Alonso P, Calzada J. Epidermoid cyst of the testis: a case report. Pediatr Radiol. 2001;31(5):365-7.

5. Price EB. Epidermoid cysts of the testis: a clinical and pathologic analysis of 69 cases from the testicular tumor registry. J Urol. 1969;102 (6):708-13. http://dx.doi.org/ 10.1016/S0022-5347(17)62236-5

6. Chams Anturi A, Cabrales Hessen M. Quiste epidermoide intratesticular. Urol Colomb. 2008;XVII(1):115-8.

7. J.S. Valla for the Group D'Etude en Urologie Pédiatrique. Testis-sparing surgery for benign testicular tumors in children. J Urol [Internet]. 2001;165(6 Pt 2):2280-3. Disponible en: http://ww w.ncbi.nlm.nih.gov/pubmed/11379598 8. Smith EA, Dillman JR. Epidermoid cyst: a rare intratesticular tumor in children. Pediatr Radiol. 2010;40(8):1450. https://doi.org/10.10 07/s00247-009-1421-4.

9. Alkhori NA, Barth RA. Pediatric scrotal ultrasound: review and update. Pediatr Radiol. 2017;47(9):1125-33.

10. Leal D, Fariña L, Pesqueira D, Meijide F, Quintana JL, Muller C, et al. Quiste epidermoide testicular. Actas Urol Esp. 2010;34(3):274-7.

11. Çakiroglu B, Sönmez NCC, Sinanoglu O, Ates L, Aksoy SHSHH, Özcan F, et al. Testicular epidermoid cyst. African J Paediatr Surg. 2015;12(1):89-90.

12. Arellano CMR, Kozakewich HPW, Diamond D, Chow JS. Testicular epidermoid cysts in children: Sonographic characteristics with pathological correlation. Pediatr Radiol. 2011;41(6):683-9.

13. Cho J-H, Chang J-C, Park B$\mathrm{H}$, Lee J-G, Son C-H. Sonographic and $\mathrm{MR}$ imaging findings of testicular epidermoid cysts. AJR Am J Roentgenol. United States; 2002 Mar;178(3):743-8.

14. Shtricker A, Silver D, Sorin E, Schreiber L, Katlowitz N, Tsivian A, et al. The value of testicular ultrasound in the prediction of the type and size 
of testicular tumors. Int Braz J Urol. 2015;41(4):655-60.

15. Pearse I, Shamberger C, Glick D, Abramson SJ, Gerald WR, Quaglia MP La. Testicular-sparing surgery for benign testicular tumors. Surgery. 1999;34(6):1000-3.

16. Caldwell BT, Saltzman AF, Maccini MA, Cost NG. Appropriateness for testis-sparing surgery based on the testicular tumor size in a pediatric and adolescent population. J Pediatr Urol. 2019;15(1):70.e1-70.e6. https://doi.or g/10.1016/j.jpurol.2018.10.011

17. Hoag NA, Afshar K, Youssef D, Masterson JST, Murphy J, MacNeily AE. Cystic intratesticular lesions in pediatric patients. J Pediatr Surg. 2013;48(8):1773-7. http://dx.doi.org/1 0.1016/j.jpedsurg.2012.10.049

18. Casaccia G, Spagnoli A, Ferro F. Simple intratesticular cysts in infancy: Testis-sparing surgery by a scrotal approach. Urology. 2000;56(5):854-5.

19. Olguner M, Akgür FMM, Aktug T, Canda T, Aktu\# T, Canda T. Testis sparing surgery for epidermoid cyst of the testis: A case report. Int Urol Nephrol. 1997;29(5):587-9.

20. Fujino J, Yamamoto H, Kisaki Y, Ishimaru Y, Uchida H, Mori Y, et al. Epidermoid cyst: Rare testicular tumor in children. Pediatr Radiol. 2004;34(2):172-4.

21. Madden-Fuentes $\mathrm{R}$, Wiener JS, Ross SS, Routh JC. Partial orchiectomy for bilateral synchronous testicular masses in a prepubescent boy: A case report. Urology. 2012;80(5):1144-6. http://dx.doi.org/1 0.1016/j.urology.2012.04.056

22. Subik MK, Gordetsky J, Yao JL, Di Sant'Agnese PA, Miyamoto $\mathrm{H}$. Frozen section assessment in testicular and paratesticular lesions suspicious for malignancy: Its role in preventing unnecessary orchiectomy. Hum Pathol. 2012;43 (9):1514-9. http://dx.doi.org/1 0.1016/j.humpath.2011.11.013

23. Tröbs RB, Krauss M, Geyer C, Tannapfel A, Körholz D, Hirsch W. Surgery in infants and children with testicular and paratesticular tumours: A single centre experience over a 25-year-period. Klin Padiatr. 2007;219(3):146-51.

24. Romo Muñoz MI, Núñez Cerezo V, Dore Reyes M, Vilanova Sánchez A, González-Peramato P, López Pereira P, et al. Testicular tumours in children: Indications for testis-sparing surgery. An Pediatr. 2018;88(5):253-8.

25. Taghavi K, Hutson JM. Testicular microlithiasis and epidermoid cysts: A common pathway? Pediatr Surg Int. 2012;28(10):1041-3.

26. Manning MA, Woodward PJ. Testicular epidermoid cysts: sonographic features with clinicopathologic correlation. J Ultrasound Med. 2010;29(5):831-7.

27. Ross JH, Kay R, Elder J. Testis sparing surgery for pediatric epidermoid cysts of the testis. J Urol. 1993;149(2):353-6. http://dx.doi.org/1 0.1016/S0022-5347(17)36080-9.

28. Neumann DPDP, Abrams GSGS, Hight DWDW. Testicular epidermoid cysts in prepubertal children: Case report and review of the world literature. J Pediatr Surg. 1997;32(12):1786-9.

29. Wu D, Shen $N$, Lin $X$, Chen X. Prepubertal testicular tumors in China: a 10-year experience with 67 cases. Pediatr Surg Int. 2018;34(12):1339-43. http://dx.doi.or g/10.1007/s00383-018-4366-6.

30. Giguere JK, Stablein DM, Spaulding JT, Mcleod DG, Paulson DF, Weiss RB. The clinical significance of unconventional orchiectomy 
approaches in testicular cancer: A report from the Testicular Cancer Intergroup Study. J Urol. 1988;139(6):1225-8. http://dx.doi.org/ 10.1016/S0022-5347(17)42873-4.

31. Ozen H, Altug U, Bakkaloglu MA, Remzi D. Significance of scrotal violation in the prognosis of patients with testicular tumours. Br J Urol. 1988;62(3):267-70. 